\title{
Interference of dissimilar photon sources
}

\author{
A. J. Bennett ${ }^{1 \star}$, R. B. Patel ${ }^{1}{ }^{2}$, C. A. Nicoll ${ }^{2}$, D. A. Ritchie ${ }^{2}$ and A. J. Shields ${ }^{1}$
}

If identical photons meet at a semi-transparent mirror they seem to leave in the same direction, an effect called 'twophoton interference'. It has been known for some time that this effect should occur for photons generated by dissimilar sources with no common history, provided the measurement cannot distinguish between the photons ${ }^{1}$. Here, we report a technique for observing such interference with isolated, unsynchronized sources for which the coherence times differ by several orders of magnitude. In an experiment we cause photons generated by different physical processes, with different photon statistics, to interfere. One of the sources is stimulated emission from a tunable laser, which has Poissonian statistics and a nanoelectronvolt bandwidth. The other is spontaneous emission from a quantum dot in a p-i-n diode ${ }^{2,3}$ with a fewmicroelectronvolt linewidth. We develop a theory to explain the visibility of interference, which is primarily limited by the timing resolution of our detectors.

Two-photon interference is at the heart of many optical quantum information processing protocols, but to be scalable these proposals require large numbers of identical photons in predetermined states. This leads to a question: what is the minimum requirement for observing interference between two sources? Since the first demonstration of two-photon interference ${ }^{4}$, the prototypical experimental arrangement has used parametric downconversion to create photon pairs by exciting a crystal with a strong laser. Experiments have been reported where a heralded photon interferes with a weak Poissonian source, ${ }^{5,6}$, although in these cases the two photon streams were derived from the same laser. Recently, interference between distinct but nominally identical downconversion sources has been demonstrated, with separate pump lasers ${ }^{7,8}$. On-demand zero-dimensional photon sources are attractive for scalable quantum information processing as they naturally create only one photon at a time ${ }^{9}$; however, care must be taken to ensure that spectral jitter and dephasing do not distinguish the photons. Emission of indistinguishable single photons was demonstrated with a semiconductor quantum $\operatorname{dot}^{10}$ and a single atom ${ }^{11}$, by optically exciting a single source two times, in quick succession. More recently, it has been shown that two identical zero-dimensional sources excited with the same laser can generate indistinguishable photons ${ }^{12-15}$. From a fundamental point of view it would be interesting to be able to cause interference of sources that are not similar. Such interference effects can be used to determine the spectral density matrix of a single photon ${ }^{6}$. Also potential applications of quantum information processing will in future require interactions between distributed sources, either for quantum computing ${ }^{16}$ or for interfacing weak lasers commonly used in quantum cryptography with the more exotic sources being investigated for quantum repeaters and quantum memories. Here, we report a demonstration of interference between non-classical emission from an electrically excited InAs quantum dot and a commercially available semiconductor laser.
To understand our experiment, it is useful to consider idealized sources incident from opposite sides on a 50/50 semi-transparent mirror (as illustrated in Fig. 1a). Two detectors at the outputs of the mirror measure the probability of two photons leaving the mirror at the same time. If we assume photons from the two sources to have perfect overlap in energy, space and time but no mutual coherence, then we need only consider the probabilities of the sources emitting a certain number of photons. The laser obeys Poissonian statistics, $\left\langle\psi_{\mathrm{a}} \mid \psi_{\mathrm{a}}\right\rangle=\exp \left(-\alpha^{2}\right) \sum_{n_{\mathrm{a}}}\left(\alpha^{2 n_{\mathrm{a}}} / n_{\mathrm{a}} !\right)\left\langle n_{\mathrm{a}} \mid n_{\mathrm{a}}\right\rangle$ and for the quantum light source $\left\langle\psi_{\mathrm{b}} \mid \psi_{\mathrm{b}}\right\rangle=(1-\eta)\left\langle 0_{\mathrm{b}} \mid 0_{\mathrm{b}}\right\rangle+\eta\left\langle 1_{\mathrm{b}} \mid 1_{\mathrm{b}}\right\rangle$. In our notation, $\eta$ and $\alpha^{2}$ are proportional to the probabilities of detecting a photon from the anti-bunched source and one from the laser, respectively, at the output of the experiment. For simplicity we expand the coherent state only up to $n_{\mathrm{a}}=2$, which is valid for a strongly attenuated laser $\alpha^{2} \ll 1$. If the sources do not interfere there are a number of ways in which the detectors can collect one photon each: either they collect both photons from the laser (with probability $2 R T \alpha^{4} / 4$ ) or they collect one photon from the laser and one from the single-photon source. This second possibility can occur if both photons are reflected (with probability proportional to $\left.R^{2} \eta \alpha^{2} / 2\right)$ or if they are both transmitted $\left(T^{2} \eta \alpha^{2} / 2\right)$. However, if the two sources are indistinguishable, then interference will occur, leading to the last two terms cancelling when $R=T$. These jointdetection probabilities $g^{(2)}(0)$ are normalized to the probability of detecting two photons at different times, $\left(\eta+\alpha^{2}\right)^{2}$. Equation (1a) gives the probability of detecting a photon in both outputs at the same time when the sources have parallel polarizations and are indistinguishable; a useful control measurement is that of data with the sources orthogonally polarized, so that the photons are entirely distinguishable and no interference occurs (1b):

$$
\begin{gathered}
g_{\|}^{(2)}(0)=\left(1+\frac{\eta}{\alpha^{2}}\right)^{-2} \\
g_{\perp}^{(2)}(0)=\left(1+\frac{2 \eta}{\alpha^{2}}\right)\left(1+\frac{\eta}{\alpha^{2}}\right)^{-2}
\end{gathered}
$$

Figure 1b plots $g_{\perp}^{(2)}(0)$ and $g_{\|}^{(2)}(0)$ versus the ratio of source intensities, $\eta / \alpha^{2}$. For $g_{\|}^{(2)}(0)$, all coincidence counts at time delay zero are due to the multiphoton emission from the laser, which falls as $\eta / \alpha^{2}$ increases. Using this simple analysis we can make the surprising prediction that the visibility of two-photon interference, $\left(g_{\perp}^{(2)}(0)-g_{\|}^{(2)}(0)\right) / g_{\perp}^{(2)}(0)$, can approach unity as $\eta / \alpha^{2}$ increases.

We now determine the coherence properties of the two sources used in our experiment. Single-photon interference measurements were carried out using a free-space Michelson interferometer with a variable time delay ${ }^{17}$ (Fig. 2a). The interference pattern, as a function of delay, is measured using an avalanche photodiode (D1). Looking at emission from the $\mathrm{X}^{-}$state of the quantum-dot source ${ }^{3}$ on its own, we see that the interference has a fringe contrast that varies as $A_{0} \exp \left(-|t| / \tau_{\text {coh }}\right)$, where $A_{0}$ is the fringe contrast at zero

${ }^{1}$ Toshiba Research Europe Limited, Cambridge Research Laboratory, 208 Science Park, Milton Road, Cambridge CB4 0GZ, UK, ${ }^{2}$ Cavendish Laboratory, Cambridge University, J J Thomson Avenue, Cambridge CB3 OHE, UK. *e-mail: anthony.bennett@crl.toshiba.co.uk. 
a

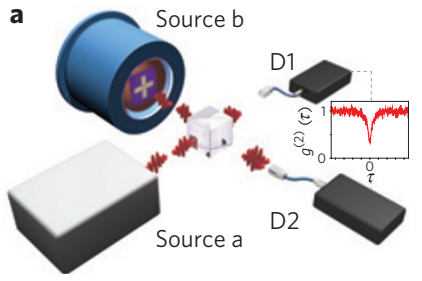

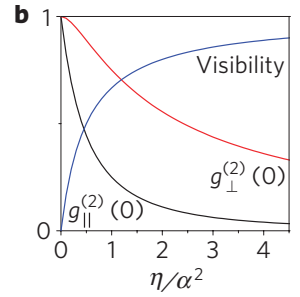

Figure 1 | Interference of a single-photon source with a weak Poissonian source. $\mathbf{a}$, Schematic of the experiment. $\mathbf{b}$, The predicted correlation function at zero time delay, $g^{(2)}(0)$, for parallel (black) and orthogonal (red) photons as a function of the intensity ratio of the sources, $\eta / \alpha^{2}$. The resulting visibility of the two-photon interference, $\left(g_{\perp}^{(2)}(0)-g_{\|}^{(2)}(0)\right) /$ $g_{\perp}^{(2)}(0)$, is shown in blue.

delay, $t$ is the delay time and $\tau_{\text {coh }}$ is the coherence time. For the dot studied here, $\tau_{\mathrm{coh}}=285 \mathrm{ps}$ at $100 \mu \mathrm{A}$. This characteristic exponential variation in contrast is an indication that the state has a Lorentzian line shape in energy of width $4.4 \mu \mathrm{eV}$. Thus, we can be sure that homogeneous processes dominate the line broadening mechanisms ${ }^{18}$. We note that the maximum fringe contrast observed at zero time delay, $A_{0}$, is below unity because of the finite spatial overlap of light that travels along the two arms of the interferometer. Separately, we have shown that the coherence time of this source is sufficient for post-selecting events of interference between successive photons emitted by the source (see Supplementary Information). The other source that we use is an external-cavity solid-state laser diode that can be tuned over several hundred microelectronvolts using a piezoelectric actuator. This source has a coherence time of $1 \mu \mathrm{s}$, which is three orders of magnitude longer than we are able to probe with our Michelson interferometer. Thus, the fringe contrast is constant at $A_{0}$ over the range of delays that we probe.

In our experiment, to obtain a finite visibility of two-photon interference these sources must have the same energy to within the sum of their linewidths ${ }^{11}$. However, our spectrometer and CCD (charge-coupled device) system have a spectral resolution of only $\sim 100 \mu \mathrm{eV}$. Hence, to ensure spectral indistinguishability we use a scheme based on single-photon interference, the layout of which is shown in Fig. 2a. Clearly the two photons have orthogonal polarization at the detectors and so will not give rise to two-photon interference. However, the single-photon interference patterns of the separate sources have a period given by their wavelength. Thus, when the two sources are detected at the same time we observe 'beating' in the intensity at the detector. The period of the beats is inversely proportional to the energy difference between the states. We have developed a simple model to illustrate how the single-photon interference fringe contrast, normalized to $A_{0}$, varies with both the energy difference between the sources and the interferometer delay (Fig. 2b). We consider only the case where the sources have the same intensity at the detectors.

In practice, we set the interferometer delay to a fixed value and measure the fringe contrast as a function of the piezovoltage applied to the laser, which results in a nearly linear variation in laser energy. As can be seen in Fig. 2c, the fringe contrast varies cosinusoidally as a function of the energy splitting between the two sources. For a delay of $380 \mathrm{ps}$, the period of the cosine variation is $34 \mu \mathrm{eV}$. With a least-squares fit to the experimental data, we can ensure degeneracy with an error estimated as below $1 \mu \mathrm{eV}$, less than the linewidth of the broader source. Using this method we have experimentally verified that the wavelengths of the sources remain stable within the accuracy of this measurement over $24 \mathrm{~h}$.

We are now able to carry out the two-photon interference experiment using the apparatus in Fig. 3a. The collinear and oppositely polarized photons are passed to an interferometer made of polarization-maintaining optic fibre. The first, polarizing,
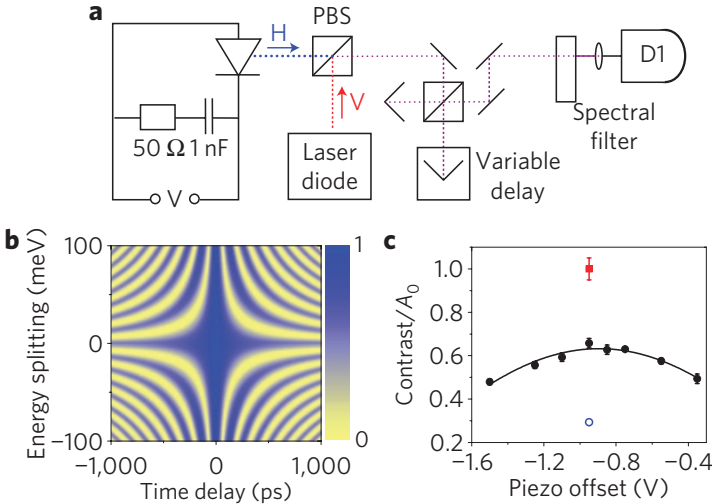

Figure 2 | Measurements of single-photon interference. a, Layout for the experiment. $\mathbf{b}$, Predicted fringe contrast as a function of the energy difference between the sources and the delay in the Michelson interferometer. c, Experimental fringe contrast for the dot and laser signals combined at 380 ps delay as the bias applied to the piezoelectric stack tunes the laser wavelength (black). Also shown are the fringe contrasts of the laser (red) and dot (blue) at the same delay, measured separately. The error bars represent standard deviations determined from least-squares fits to the data.

coupler $\mathrm{C}_{\mathrm{A}}$ ensures that every photon from the dot takes the upper path to the final, non-polarizing, 50/50 coupler $C_{B}$ and that every photon from the laser takes the lower path. This design increases the probability of the two photons reaching the final coupler from opposite sides by a factor of four, relative to previous experiments ${ }^{10}$. Correlations at the outputs of $\mathrm{C}_{\mathrm{B}}$ are measured with two silicon avalanche photodiodes. A half-wave plate in the path taken by the laser photon switches its polarization between being parallel and orthogonal to the quantum dot's photon every few minutes. This enables us to build up the correlations for the cases where the photons are and are not interfering within the same integration time. Thus, any slow drift in the position of the sources, or the fibres, which might change the ratio of their intensities at the detectors, is averaged out between pairs of measurements. During the course of each measurement, the ratio of intensities is stable to within $5 \%$.

Figure 3d,e shows experimental data recorded for equalintensity sources. The measurement of $g_{\perp}^{(2)}(\tau)$ shows a dip at time zero due to the anti-bunched nature of the quantum light source, as expected. More strikingly, we can see a clear difference between the measurements for parallel and orthogonal polarizations, which is a result of two-photon interference. This finite visibility constitutes the main result of our experiment and occurs due to interference between photons from the weak laser and the anti-bunched source, despite their different linewidths and lack of common history.

To further quantify this result, a full analysis, including non-ideal source parameters, enables us to calculate the correlation as a function of time (equation (2)):

$$
\begin{aligned}
g_{\phi}^{(2)}(\tau)= & R_{\mathrm{f}}(\tau) \\
& \otimes\left[\frac{2 \eta \alpha^{2}\left(1-\gamma^{2} \cos ^{2}(\phi) \exp \left(\frac{-|\tau|}{\tau_{\mathrm{coh}}}\right)\right)+\left(\eta^{2} g_{\mathrm{HBT}}^{(2)}(\tau)+\alpha^{4}\right)}{\left(\eta+\alpha^{2}\right)^{2}}\right]
\end{aligned}
$$

where $\phi$ is the angle between the polarizations of the two photons, $\gamma=\left\langle\psi_{\mathrm{a}} \mid \psi_{\mathrm{b}}\right\rangle$ is a measure of the overlap of the wavefunctions of the two photons, $R_{\mathrm{f}}$ is the detection system response function and $R=T$. We note that in the case where $g_{\mathrm{HBT}}^{(2)}(0)=0, \gamma=1$ and $\tau=0$, this reverts to the form given in equations (1a) and (1b), as expected. In this experiment, $\eta$ and $\alpha^{2}$ are of the order of $10^{-3}$. Separately we measure the photon statistics of our sources using 


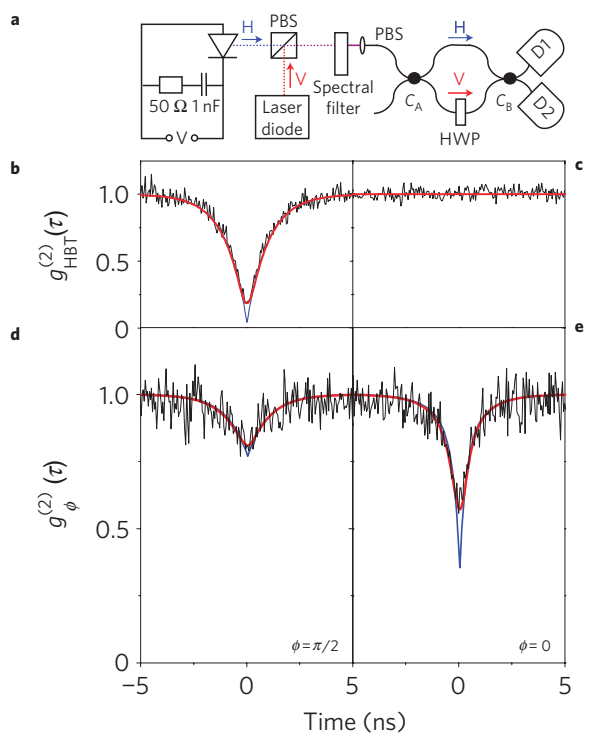

Figure 3 | Measurements of photon statistics. a, Experimental layout for two-photon interference between the sources. b-e, Intensity correlation functions recorded for the quantum light source alone (b) and the laser alone (c), with both sources having orthogonal (d) and parallel (e) polarizations. These plots show the measured data (black), and predicted correlations for infinitely fast detectors (blue) and for the measured detector response function (red).

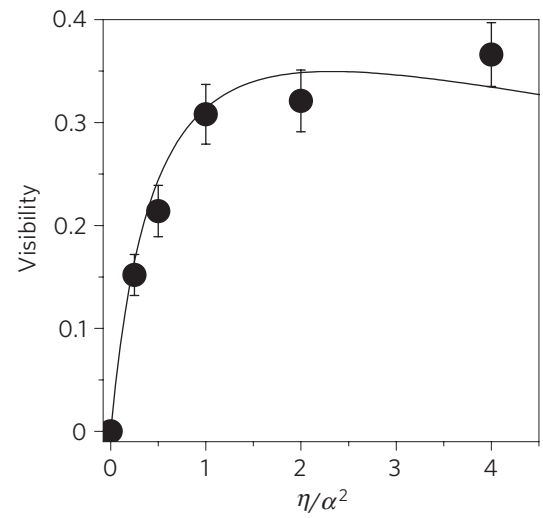

Figure 4 | Measurement of the visibility of two-photon interference as a function of $\eta / \alpha^{2}$. Included as a solid line is a fit using the known detector response time and $\gamma=91 \%$. The error bars represent standard deviations determined from least-squares fits to the data.

a Hanbury-Brown and Twiss (HBT) arrangement (equivalent to that of Fig. 3a, with only one source operational at a time). For the laser, the autocorrelation function $g_{\mathrm{HBT}}^{(2)}(\tau)=1$ (Fig. 3c), as would be expected for a photon source with Poissonian statistics. For the quantum-dot source we expect anti-bunched emission with a dip at time zero. We can predict the precise shape of this autocorrelation $^{18,19}$ using the independently measured radiative lifetime ( $985 \mathrm{ps})$, the contribution from background and dark counts (which sum to 0.04 of the signal from the quantum state) and the resolution of our detection system (a Gaussian with width $428 \mathrm{ps})$. This model suggests $g_{\mathrm{HBT}}^{(2)}(0)=0.19$, which is consistent with our experimental measurement (Fig. 3b). From these parameters we calculate $g_{\perp}^{(2)}(\tau)$ and $g_{\|}^{(2)}(\tau)$ for equal-intensity sources being mixed. The correlations that would be observed for infinitely fast detectors are shown as blue lines in Fig. 3d,e. However, when the response function of the detection system is included, we obtain the curves shown in red. The only free parameter is the wavefunction overlap $\gamma=0.91$.
A series of measurements was made of the visibility of the interference as a function of the ratio of the intensities of the two sources, $\eta / \alpha^{2}$. Figure 4 shows the data and our prediction for $\gamma=0.91$. From our theory we predict that maximum visibility will be observed for $\eta / \alpha^{2} \sim 2$, which is a result of the finite width of $R_{\mathrm{f}}$. The agreement between theory and experiment is good. It is remarkable that we can infer such a high overlap of the photons given the fundamental differences in the sources. We note that the maximum measured visibility of interference is set by the ratio of the coherence time of the solid-state source to the response time of the detection system. In future, the raw visibility could be increased by using superconducting detectors with a fast response time or atomic sources with a long coherence time.

Received 6 March 2009; accepted 14 July 2009; published online 30 August 2009

\section{References}

1. Mandel, L. Photon interference and correlation effects produced by independent quantum sources. Phys. Rev. A 28, 929-943 (1983).

2. Yuan, Z. L. et al. Electrically driven single-photon source. Science 295 , 102-105 (2002).

3. Bennett, A. J. et al. Microcavity single-photon-emitting diode. Appl. Phys. Lett. 86, 181102 (2005)

4. Hong, C. K., Ou, Z. Y. \& Mandel, L. Measurement of subpicosecond time intervals between two photons by interference. Phys. Rev. Lett. 59, 2044-2046 (1987).

5. Rarity, J. G., Tapster, P. R. \& Loudon, R. Non-classical interference between independent sources. J. Opt. B 7, 171-175 (2005).

6. Wasilewski, W., Kolenderski, P. \& Frankowski, R. Spectral density matrix of a single photon measured. Phys. Rev. Lett. 99, 123601 (2007).

7. Kaltenbaek, R. et al. Experimental interference of independent photons. Phys. Rev. Lett. 96, 240502 (2006).

8. Halder, M. et al. Entangling independent photons by time measurement. Nature Phys. 3, 692-695 (2007).

9. Lounis, B. \& Orrit, M. Single-photon sources. Rep. Prog. Phys. 68, 1129-1179 (2005).

10. Santori, C., Fattal, D., Vučković, J., Solomon, G. S. \& Yamamoto, Y. Indistinguishable photons from a single-photon device. Nature 419, 594-597 (2002).

11. Legero, T., Wilk, T., Hennrich, M., Rempe, G. \& Kuhn, A. Quantum beat of two single photons. Phys. Rev. Lett. 93, 070503 (2004).

12. Beugnon, J. et al. Quantum interference between two single photons emitted by independently trapped atoms. Nature 440, 779-782 (2006).

13. Maunz, P. et al. Quantum interference of photon pairs from two remote trapped atomic ions. Nature Phys. 3, 538-541 (2007).

14. Chaneliere, T. et al. Quantum interference of electromagnetic fields from remote quantum memories. Phys. Rev. Lett. 98, 113602 (2007).

15. Sanaka, K., Pawlis, A., Ladd, T., Lischka, K. \& Yamamoto, Y. Indistinguishable photons from independent semiconductor nanostructures. Phys. Rev. Lett. 103, 053601 (2009).

16. Lim, Y. L., Beige, A. \& Kwek, Y. C. Repeat-until-success linear optics distributed quantum computing. Phys. Rev. Lett. 95, 030505 (2005).

17. Kammerer, C. et al. Interferometric correlation spectroscopy in single quantum dots. Appl. Phys. Lett. 81, 2737-2739 (2002).

18. Patel, R. B. et al. Postselective two-photon interference from a continuous nonclassical stream of photons emitted by a quantum dot. Phys. Rev. Lett. 100, 207405 (2008).

19. Michler, P. et al. Quantum correlation among photons from a single quantum dot at room temperature. Nature 406, 968-970 (2000).

\section{Acknowledgements}

This work was partly supported by the EU through the IST FP6 Integrated Project Qubit Applications (QAP: contract number 015848). EPSRC provided support for R.B.P. and QIPIRC for C.A.N.

\section{Author contributions}

R.B.P., C.A.N. and D.A.R. designed and fabricated the samples. A.J.B. and R.B.P. carried out the optical experiments. A.J.S. guided the work. A.J.B. wrote the manuscript, with input from the coauthors.

\section{Additional information}

Supplementary information accompanies this paper on www.nature.com/naturephysics. Reprints and permissions information is available online at http://npg.nature.com/ reprintsandpermissions. Correspondence and requests for materials should be addressed to A.J.B. 